\title{
Antisocial Personality Disorder Among Patients in Treatment for Alcohol Use Disorder (AUD): Characteristics and Predictors of Early Relapse or Drop-Out
}

\author{
Susmita Pandey (iD) ${ }^{1,2}$ \\ Ingeborg Bolstad' \\ Lars Lien ${ }^{1,3}$ \\ Jørgen G Bramness ${ }^{1,4,5}$ \\ 'Norwegian National Advisory Unit on \\ Concurrent Substance Abuse and Mental \\ Health Disorders, Innlandet Hospital Trust, \\ Brumunddal, Norway; ${ }^{2}$ Institute of Clinical \\ Medicine, University of Oslo, Oslo, Norway; \\ ${ }^{3}$ Department of Health and Social Science, \\ Innlandet University of Applied Science, \\ Elverum, Norway; ${ }^{4}$ Department of Clinical \\ Medicine, UiT - Norway's Arctic University, \\ Tromsø, Norway, ${ }^{5}$ Norwegian Institute of \\ Public Health, Oslo, Norway
}

Background: Patients with alcohol use disorders (AUD) vary significantly in many clinically important characteristics making them a heterogenous group. AUD patients with comorbid antisocial personality disorder (ASPD) form an important sub-group, and studies indicate that these patients may have poorer treatment outcomes. Therefore, we aimed to investigate the characteristics of AUD inpatients with comorbid ASPD and identify predictors of early relapse or treatment drop-out in these patients.

Methods: In a longitudinal study of AUD patients ( $\mathrm{n}=113 ; 30$ females; aged 27 to 72 years) in treatment at three residential rehabilitation clinics in Norway, we used interviews and self-report questionnaires to collect data on alcohol use, mental health, and trauma experience. In addition, we assessed biochemical parameters. The patients were followed up at 6 weeks to identify early relapse or drop-out.

Results: Prevalence of ASPD among AUD patients was 15\%. AUD patients with comorbid ASPD were exclusively male, of younger age, and reported more childhood trauma, and adult attention-deficit-hyperactivity-disorder symptoms. They reported more hazardous drinking behavior and more often had dependence on substances in addition to alcohol. The presence of ASPD did not predict early relapse or drop-out. However, early relapse or drop-out in ASPD patients was associated with childhood and adult trauma, younger age of drinking debut, and higher baseline prolactin levels.

Conclusion: AUD patients with ASPD had different clinical characteristics to other AUD patients and they had specific predictors of early relapse or drop-out. Our findings indicate that the early relapse or drop-out among AUD patients with ASPD may be attributed to environmental and possibly biological vulnerability. However, further studies with larger sample size are warranted to confirm these preliminary associations.

Keywords: alcohol use disorder, antisocial personality disorder, relapse, drop-out

\section{Introduction}

Alcohol use disorder (AUD) is a major public health issue. Harmful use of alcohol causes 1 in 20 deaths worldwide. ${ }^{1}$ A systematic analysis for the global burden of disease revealed that alcohol use is the seventh leading risk factor for deaths and loss of disability-adjusted life-years (DALYs). ${ }^{2}$ AUD is the most common type of substance use disorder in Norway $^{3}$ with a lifetime prevalence of around seven per cent. ${ }^{4}$ Several effective therapeutic interventions in the treatment of AUD are
Correspondence: Susmita Pandey Email susmita.research@gmail.com 
available $^{5,6}$ and those who receive professional help for AUD have better outcome than those who try to recover on their own. ${ }^{7,8}$ However, the uptake of treatment is low. ${ }^{4,9}$

More concerning, even after receiving treatment, more than half of patients relapse within a year. ${ }^{10,11}$ Relapse can be attributed to several psychosocial and biological factors. Among others, marital status, ${ }^{12}$ traumatic experiences, ${ }^{13}$ psychological distress, ${ }^{14}$ and presence of co-existing psychopathology including anxiety, depression, ${ }^{15}$ and attention deficit hyperactivity disorder $(\mathrm{ADHD}){ }^{16}$ may influence relapse. Moreover, relapse may be attributed to alcohol-induced neuroadaptation such as alteration in the brain dopamine system. ${ }^{17,18}$ Prolactin, to some extent, reflects the activity of the central nervous dopamine system as dopamine is a major inhibitor of prolactin release. ${ }^{19}$ Furthermore, it has been found that the increase in the level of prolactin is associated with more craving ${ }^{20}$ which is in turn associated with relapse. ${ }^{21}$

Drop-out is another substantial hindrance in AUD treatment. ${ }^{22}$ Drop-out rates from alcohol treatment programs have been reported to be over $50 \% .^{23}$ Drop-out is related to several factors such as lack of motivation for treatment ${ }^{24}$ personality factors like lower cooperativeness, lower persistence, impulsivity, ${ }^{25}$ and co-existing psychopathology. ${ }^{26}$ Tools that identify patients at risk are being researched pivotally so that drop-out from treatment can be prevented. ${ }^{27}$ There is an unequivocal understanding that the entire AUD population cannot be treated as a homogenous group. Therefore, endophenotype-focused interventions could be beneficial in minimizing relapse or drop-out. $^{28}$

An important endophenotype of AUD are patients with comorbid antisocial personality disorder (ASPD). ${ }^{29}$ ASPD is characterized by a life-long pattern of explicit antisocial acts with traits of impulsivity, irritability and remorselessness that begin in childhood. ${ }^{30}$ Although a highly prevalent personality disorder ${ }^{31}$ these patients seldom seek treatment ${ }^{32}$ and if they do, their chances of adhering to the treatment are low because of their tendency to violate rules. $^{33}$

AUD is highly prevalent in individuals with ASPD, with figures reaching up to 3 in $4 .{ }^{34}$ The comorbidity may be a result of overlapping genetic and environmental underpinnings. ${ }^{35}$ Shared environmental risk factors such as childhood trauma, coercive parenting and antisocial peer affiliation is associated with AUD-ASPD comorbidity. ${ }^{36}$ Heritable personality traits like impulsivity, novelty-seeking and negative emotionality, common in ASPD, incapacitate one's ability to ignore the immediate reward offered by alcohol. This jeopardizes the ability to inhibit habitual use of alcohol regardless of the significant psychological and physical distress it causes, leading to the genesis of AUD. ${ }^{29}$ Conversely, excessive alcohol consumption may lead to neuroadaptation that results in increased impulsivity or negative emotionality as seen in ASPD.

Comorbid ASPD is associated with greater AUD severity ${ }^{37}$ and represents a special challenge in AUD treatment. The comorbidity leads to poorer treatment outcomes ${ }^{38,39}$ thereby increasing the chance of relapse or drop-out $^{25,40}$ and hence warrants a tailored treatment. Thus, it is important to identify the unique factors that predicts relapse or drop-out in AUD population with comorbid ASPD so that they could be targeted during the treatment. The aim of this study is therefore to investigate the characteristics of AUD patients with comorbid ASPD under residential treatment for AUD and identify the predictors of early relapse or drop-out in these patients. Against this background, our hypothesis is twofold.

1. AUD patients with comorbid ASPD are different from those without comorbid ASPD.

2. There are specific factors associated with early relapse or drop-out in AUD patients with ASPD.

\section{Materials and Methods}

\section{Study Participants}

The study participants were patients receiving treatment for AUD ( $\mathrm{n}=113 ; 30$ females) at three different residential rehabilitation clinics in Norway. These rehabilitation clinics employed abstinence-oriented rehabilitative treatment including medications, cognitive behavioral therapy, and group therapy. The treatment programs ranged from 3 to 9 months. At the time of study enrollment, the participants had been receiving inpatient treatment for (median, 25-75 percentiles) 7, 5-12 days and reported being abstinent for (median, 25-75 percentiles) 19, 13-30 days. The patients were between the age of 27 to 72 years. They were followed up at 6 weeks from the baseline. The study was approved by the Norwegian Regional Ethics Committee (Reference number 2017/ 1314). The research complied with the Helsinki Declaration. Written informed consent was obtained from the patients before enrollment into the study. 


\section{Inclusion and Exclusion Criteria}

The inclusion criteria were age of 18 years and older, familiarity with a Scandinavian language, currently receiving inpatient treatment for AUD, self-report of minimum of 5 days of abstinence before enrollment into the study, and intention to participate in the follow-up at 6 weeks from baseline. The patients who were deemed unable to provide informed consent or safely participate in the study by clinical staff because of psychosis, cognitive impairment, or severe somatic illness were excluded from the study. Furthermore, the patients from whom a blood sample could not be drawn from superficial veins of arm were not included in the study.

\section{Measures}

\section{Baseline Assessment}

Mini International Neuropsychiatric Interview

Mini International Neuropsychiatric Interview (M.I.N.I) Norwegian translation version 6.0 was used to screen ASPD and dependence on substances other than alcohol. M.I.N.I 6.0 is a short structured diagnostic interview for DSM-IV psychiatric disorders. The ASPD module of the M.I.N.I is comprised of 12 questions about antisocial behavior and acts with precision between those exhibited before and after the age of 15 years. Dependence on substances other than alcohol was identified using the non-alcohol psychoactive substance use disorders module of the M.I. N.I. This module gives the diagnosis of current substance dependence and current substance abuse by asking questions about use of psychoactive substances within the past 12 months. The diagnosis of either current substance dependence or current substance abuse was considered as dependence on substances other than alcohol in our study.

\section{Biological Measures Related to Alcohol Use and Their Analysis}

Phosphatidylethanol (PEth; 16:0/18:1), a direct marker of alcohol consumption was examined to identify relapse. In addition, other biological measures related to alcohol use Gamma-glutamyltransferase $(\gamma-\mathrm{GT})$ and prolactin were also examined.

Venous blood was drawn from the median cubital vein in serum gel tubes for $\gamma-\mathrm{GT}$ and prolactin and in ethylenediaminetetraacetic acid (EDTA) tubes for PEth. Immediately after the sample collection, the tubes were turned upside down about 8 to 10 times. They were then set to rest in a blood tube stand. After 30-60 minutes, the serum gel tubes were centrifuged to separate the serum.
The blood samples were then transferred to the laboratory to run the required analysis.

PEth was measured by supercritical fluid chromatographymass spectrometry (SFC-MS) using ACQUITY Ultraperformance Convergence Chromatography ( $\mathrm{UPC}^{2}$ ) system (Waters, Milford, MA, USA). The analytical coefficient of variation of the assay was $8.0 \%$ and the detection limit was $0.03 \mu \mathrm{mol} / \mathrm{L}$.

\section{Psychological Distress Measure}

The Norwegian version of Hopkins Symptom checklist (HSCL) with 10 items, previously used in an AUD population, was used to identify general mental distress. ${ }^{41}$ The questions in HSCL-10 asked the respondents to identify their experience of symptoms related to anxiety and depression over the past week on a scale of 1 (not at all) to 4 (extremely). The mean score was calculated producing a range of scores from 1 to 4 where higher score corresponds to more psychological distress. A study reported that the reliability of the Norwegian version of HSCL-10 as tested by Cronbach's Alpha was $0.88 .^{42}$ In the current study, it was 0.90 .

\section{Accounts of Trauma}

Accounts of trauma were assessed using a structured selfreport questionnaire with five questions. This questionnaire has been used previously to interview a psychiatric population. ${ }^{37}$ The first three items asked about traumatic experiences in childhood which included sexual assaults, physical abuse and other significant traumatic events (3) while the last two items asked about traumatic experiences in adulthood which included sexual assaults or physical abuse (4) and other significant traumatic events (5). For each item, the patients could choose: No, Yes, once or Yes, several times. The sum score indicating severity of trauma was calculated separately for childhood and adulthood resulting in a range of score from 0 to 6 for childhood trauma and 0 to 4 for adulthood trauma.

\section{The Adult Attention Deficit Hyperactivity Disorder Self-Report Scale}

Symptoms of ADHD were assessed using World Health Organization (WHO) Adult ADHD Self-Report Scale (ASRS). ${ }^{43}$ It is an 18-item questionnaire based on the Diagnostic and Statistical Manual of Mental Disorders fourth edition text revision (DSM-IV-TR) criteria. We used the six-item screening version of the ASRS that has demonstrated better specificity and sensitivity than the full version. ${ }^{44}$ It is scored in a 5 -point Likert scale ranging 
from Never to Very often (4). The sum score was calculated producing a range of score from 0 to 24 where higher score corresponds to more ADHD symptoms. A study reported that the reliability of Norwegian version of ASRS- 6 as measured by Cronbach's Alpha was 0.64 in men and 0.72 in women. ${ }^{45}$ In our study, for the mixed sample of men and women, it was 0.80 .

\section{Self-Report Measures on Addiction}

The WHO developed Alcohol Use Disorders Identification test (AUDIT) was used to screen hazardous alcohol consumption. ${ }^{46}$ It consists of 10 -questions about experience with alcohol within the last year. Each response has scores that range from 0 to 4 which total to a score between 0 and 40. A higher score indicates more hazardous alcohol use. A study reported that the reliability of the Norwegian version of AUDIT as measured by Cronbach's Alpha was $0.78 .^{47}$ In our study, it was 0.77 . In addition to AUDIT, we collected information on patients' age (years) at first alcohol use and history of previous AUD treatment.

\section{Follow-Up Assessment}

The patients were followed up 6 weeks after the baseline assessment. Their level of PEth in the blood sample was measured. In addition, the patients who had dropped out of the treatment program before the 6-week follow-up were identified.

\section{Definition of Early Relapse and Drop-Out Early Relapse}

Early relapse was defined based on changes in the PEth values. The PEth concentration at the beginning of abstinence influences the time taken for elimination. ${ }^{48}$ We thus calculated the relative decrease in PEth from the baseline to the 6-week follow-up by using the formula (PEth at baseline - PEth at 6 weeks)/(PEth at baseline). PEth generally is considered to have terminal elimination half-life $\left(T_{1 / 2}\right)$ of around 6 days but some studies report a $T_{1 / 2}$ of up to 12 days. ${ }^{49}$ The elimination time is usually as long as five $\mathrm{T}_{1 / 2}^{50}$ and taking the highest figure for $T_{1 / 2}$ into consideration, a duration of 60 days (five $T_{1 / 2}$ of 12 days) would be required to achieve $100 \%$ relative change in PEth from baseline. In this early follow-up at 42 days, we would expect that the relative change in PEth should be at least $70 \%$. There were seven patients with less than $70 \%$ decrease in PEth values from the baseline to the follow-up. In addition, one patient had an increased level of PEth at the 6-week follow-up, resulting in a total of eight relapse cases.

\section{Drop-Out}

Patients who discontinued their treatment and left the rehabilitation clinics before completion of the planned course of treatment were defined as drop-out. Before the 6-week follow-up, 12 patients dropped out of treatment and they were identified as early dropouts.

Studies suggest that one of the main reasons for treatment drop-out is a severe desire to use the substance indicating that treatment drop-out is closely related to relapse. ${ }^{51}$ We compared the drop-out group with the relapse group and found they did not differ significantly in any variables that were measured including background variables, psychosocial variables, and both self-report and biological variables related to alcohol use. Hence, they were considered as a single group for analysis and referred as relapse or drop-out group hereafter.

\section{Statistical Analyses}

Statistical analyses were performed using SPSS version 23.0 for Windows. Descriptive statistics were used to present the characteristics of the groups with and without ASPD and to show the differences in early relapse or drop-out pattern. The values were not normally distributed and were therefore presented as medians and 25-75 percentiles in the tables. Fisher's exact test for categorical variables and Mann Whitney's $U$-test for continuous variables were used for comparison between groups. The effect sizes were reported as odds ratios (OR) for categorical variables and as " $r$ " for the continuous variables. Cohen's guidelines were used for interpretation of " $r$ " where 0.5 and above is a large effect, 0.3 is a medium effect, and 0.1 is a small effect. ${ }^{52}$ Furthermore, logistic regression was performed to identify factors that could be characteristic of ASPD comorbidity among AUD patients and OR along with 95\% confidence intervals (CI) were reported. All significant covariates from the unadjusted analysis were included in the adjusted logistic regression model. The correlation between all covariates was $<0.7$. Regression analysis to predict relapse or dropout could not be done due to small sample size in the subgroups. Statistical tests were two-tailed with a significance level of $\alpha=0.05$.

\section{Results \\ Difference Between AUD Patients with and without Comorbid ASPD}

Table 1 presents the differences in characteristics between patients with and without ASPD ( $n=17$ and $n=96$, respectively). The patients with comorbid ASPD were exclusively 
Table I Differences Between Patients with and without Antisocial Personality in a Cohort of Patients in Treatment for Alcohol Use Disorder $(n=113)$

\begin{tabular}{|c|c|c|c|c|}
\hline & $\begin{array}{l}\text { AUD Patients without ASPD } \\
\qquad n=96(85 \%)\end{array}$ & $\begin{array}{l}\text { AUD Patients with ASPD } \\
\qquad n=17(15 \%)\end{array}$ & $p$-values & OR or $r$ \\
\hline \multicolumn{5}{|l|}{ Background } \\
\hline Gender (male) & $66(68.8)$ & $17(100)$ & $0.006^{\mathrm{a}}$ & \\
\hline Age (years) & $54.1(48.3-58.5)$ & $39.8(32.7-50.9)$ & $<0.00 \mathrm{I}^{\mathrm{b}}$ & 0.35 \\
\hline Having paid job as source of income & $15(20.0)$ & $2(16.7)$ & $1.000^{\mathrm{a}}$ & 0.80 \\
\hline Marital status (living with a partner) & $21(27.6)$ & I (8.3) & $0.281^{\mathrm{a}}$ & 0.24 \\
\hline \multicolumn{5}{|l|}{ Psychosocial measures } \\
\hline HSCL (mean score) & $2.0(1.5-2.6)$ & $2.2(1.9-2.5)$ & $0.288^{b}$ & 0.12 \\
\hline Childhood trauma severity & $2.0(0.0-3.0)$ & $4.0(2.3-4.8)$ & $0.003^{b}$ & 0.32 \\
\hline Adult trauma severity & I.0 (0.0-2.0) & $2.0(0.5-2.0)$ & $0.104^{b}$ & 0.18 \\
\hline ASRS 6 (sum score) & $11.0(9.0-16.0)$ & $16.0(13.5-18.0)$ & $0.009^{b}$ & 0.29 \\
\hline \multicolumn{5}{|l|}{ Self-report measures on addiction } \\
\hline Age at first alcohol use (years) & $15.0(14.0-16.0)$ & $14.0(\mid 2.0-15.0)$ & $0.05 I^{b}$ & 0.18 \\
\hline AUDIT & $29.0(21.5-33.5)$ & $34.0(28.3-38.7)$ & $0.022^{\mathrm{b}}$ & 0.25 \\
\hline History of previous AUD treatment & $62(65.3)$ & $12(70.6)$ & $0.785^{\mathrm{a}}$ & 1.28 \\
\hline Dependence other substances & $14(14.6)$ & $10(62.5)$ & $<0.001^{a}$ & 9.76 \\
\hline \multicolumn{5}{|c|}{ Biological measures for alcohol use at inclusion } \\
\hline$\gamma-\mathrm{GT}(\mathrm{U} / \mathrm{L})$ & $42.5(26.0-77.5)$ & $29.0(20.5-63.0)$ & $0.057^{b}$ & 0.18 \\
\hline PEth $(\mu \mathrm{mol} / \mathrm{L})$ & $0.3(0.1-0.6)$ & $0.2(0.0-0.6)$ & $0.147^{b}$ & 0.14 \\
\hline Prolactin (mU/L) & $133.5(99.0-186.5)$ & $132.0(110.0-186.5)$ & $0.753^{b}$ & 0.03 \\
\hline \multicolumn{5}{|l|}{ Relapse or drop-out 6 weeks } \\
\hline Relapsed or dropped out at 6 weeks & $17(19.5)$ & $3(21.4)$ & $1.000^{\mathrm{a}}$ & 1.04 \\
\hline
\end{tabular}

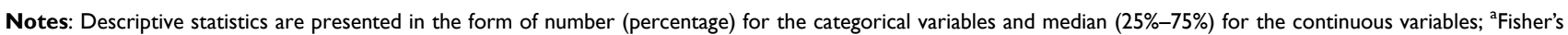
exact test; 'Mann Whitney's U-test; effect sizes are reported as Odds ratio (OR) for categorical variable and as " $r$ " for continuous variables.

Abbreviations: ASPD, antisocial personality disorder; AUD, alcohol use disorder; HSCL, Hopkins' Symptom Checklist; ASRS, Adult ADHD Self-Report Scale; AUDIT, Alcohol Use Disorder Identification Test; PEth: Phosphatidylethanol and $\gamma$-GT: Gamma-glutamyltransferase.

males while those without ASPD included $30(31.2 \%)$ females. The median age of patients with ASPD was 39.8 (25-75 percentiles: 32.7-50.9) years which was lower than that of patients without ASPD who had a median age of 54.1 (48.3-58.5) years $(r=0.35, p<0.001)$. The patients with ASPD had experienced more childhood trauma $(r=0.32$, $p=0.003)$ and had more adult ADHD symptoms $(r=0.29$, $p=0.009)$. Their median age of drinking debut was 14.0 $(12.0-15.0)$ years while that of patients without ASPD was $15.0(14.0-16.0)$ years $(r=0.18, p=0.051)$. The patients with ASPD had more hazardous drinking behavior $(r=0.25, p=$ 0.022 ) and they more often met criteria for dependence on substances other than alcohol $(\mathrm{OR}=9.76, p<0.001)$. However, there was no significant difference in the early relapse or drop-out rate between the personality groups at 6 week follow-up; $19.5 \%$ among the patients without ASPD and $21.4 \%$ among the patients with $\operatorname{ASPD}(\mathrm{OR}=1.04, p=1.000)$.
Table 2 shows the results from the logistic regression model $(\mathrm{n}=113)$ of having comorbid ASPD using variables that were significant $(p<0.05)$ in Table 1. A younger age (OR $=0.894,95 \%$ CI: $0.806-0.992)$ and higher childhood trauma severity $(\mathrm{OR}=2.019 ; 1.097-3.717)$ increased the odds of ASPD comorbidity when adjusted for one another along with ADHD symptoms, hazardous drinking behavior, and dependence on substances other than alcohol.

\section{Factors Associated with Early Relapse or Drop-Out in AUD Patients with Comorbid ASPD}

Table 3 shows the specific factors related to early relapse or drop-out in AUD patients with ASPD. In patients with comorbid ASPD, those who relapsed or dropped out had more psychological distress $(r=0.69, p=0.040)$ and had 
Table 2 Logistic Regression Model of Having Comorbid Antisocial Personality Disorder Among Patients in Treatment for Alcohol Use Disorder $(n=113)$

\begin{tabular}{|l|c|c|c|c|c|c|}
\hline & Reference & & Unadjusted & p-values & Adjusted a) & p-values \\
\hline Age & Continuous & OR $(95 \% \mathrm{Cl})$ & $0.89(0.84-0.95)$ & $<0.001$ & $0.89(0.81-0.99)$ & 0.035 \\
Childhood trauma & Continuous & OR $(95 \% \mathrm{Cl})$ & $1.63(1.15-2.30))$ & 0.006 & $2.02(1.10-3.72)$ & 0.024 \\
severity & & & & & \\
ASRS 6 (sum score) & Continuous & OR $(95 \% \mathrm{Cl})$ & $1.23(1.04-1.46)$ & 0.014 & $1.31(0.95-1.79)$ & 0.096 \\
AUDIT (sum score) & Continuous & OR $(95 \% \mathrm{Cl})$ & $1.15(1.01-1.30)$ & 0.033 & $1.07(0.91-1.25)$ & 0.413 \\
Dependence other & Absence of other substance & OR $(95 \% \mathrm{Cl})$ & $9.76(3.06-31.14)$ & $<0.001$ & $4.38(0.62-30.78)$ & 0.137 \\
substances & dependence & & & & & \\
\hline
\end{tabular}

Notes: ${ }^{\text {a) }}$ Adjusted for all significant covariates in the unadjusted analysis; Nagelkerke's $\mathrm{R}^{2}$ for the multiple regression analysis was 0.577 and the Hosmer-Lemeshow goodness of fit test was $\chi^{2}=9.623, d f=8, p=0.292$.

Abbreviations: OR, odds ratio; $\mathrm{Cl}$, confidence Interval; ASRS, Adult ADHD Self-Report Scale; AUDIT, Alcohol Use Disorder Identification Test.

experienced more childhood and adulthood trauma $(r=$ $0.70, p=0.036$ and $r=0.76, p=0.023$, respectively). The comparison of relapse or drop-out patients across the personality groups showed that the ASPD patients who relapsed or dropped out experienced more childhood and adult trauma ( $r=0.56, p=0.35$ and $r=0.56, p=0.037$, respectively), were younger when they had their first experience with alcohol $(r=0.53, p=0.019)$, measured lower on the level of $\gamma$-GT $(r=0.46, p=0.044)$, and higher on the level of prolactin $(r=0.51, p=0.025)$ when compared to the patients without ASPD who relapsed or dropped out. Figure 1 illustrates the differences in relapse or drop-out pattern between the personality groups in terms of age of drinking debut, severity of childhood and adult trauma, and level of baseline prolactin.

\section{Discussion}

In this longitudinal study of AUD patients in treatment, the first hypothesis that the AUD patients with comorbid antisocial personality disorder (ASPD) are different from those without comorbid ASPD was confirmed. We found that the patients with ASPD were males, of younger age, reported more childhood trauma, and more adult ADHD symptoms. In addition, patients with ASPD had more hazardous drinking behavior, and they more often met criteria for dependence on substances other than alcohol. Comorbid ASPD in AUD did not predict early relapse or drop-out in this study. In line with our second hypothesis, we found some specific predictors of early relapse or dropout in AUD patients with ASPD. Their relapse or drop-out was more often associated with traumatic experiences, younger age of drinking debut, and higher prolactin levels at baseline.
The observed male predominance in ASPD is in line with other studies. ${ }^{53}$ This can also be drawn from the typologies-focused research in AUD. Among two types of AUD patients identified by Cloninger and colleagues, Type II AUD patients exhibited antisocial behaviors and were predominantly men. ${ }^{54}$ Our finding of younger age among AUD patients with comorbid ASPD corresponds with "young antisocial subtype of alcohol dependence". 55 Moss and colleagues identified five clusters of AUDs in a nationally representative sample from the United States. Of the five clusters, cluster 4; the young antisocial subtype was described, among other things, as a subtype with higher probability of having ASPD and a relatively young age. Also, AUD patients with comorbid ASPD are more likely to seek treatment at a younger age ${ }^{56}$ which is partially reflected in our data because we found that the ASPD patients were in treatment at a younger age for the current episode of inpatient treatment. However, we do not have information on the age at which the patients first sought treatment for AUD to confirm the finding of Megan and colleagues. We also found that AUD patients with ASPD had more adult ADHD symptoms and had experienced more childhood trauma, both of which are consistent with previous studies. ${ }^{57-60}$ Furthermore, we found that the AUD patients with ASPD had more hazardous drinking behavior which also corresponds to earlier findings. ${ }^{61}$ Antagonism and disinhibition predict hazardous alcohol use ${ }^{62}$ and antisocial behavior is associated with increased lifetime alcohol problems. ${ }^{63}$ Moreover, we found that AUD patients with ASPD more often met criteria for dependence on substances other than alcohol. In line with our findings, other studies report that comorbidity with other substance dependence increases with ASPD severity. ${ }^{61}$ All these findings suggest that antisocial 


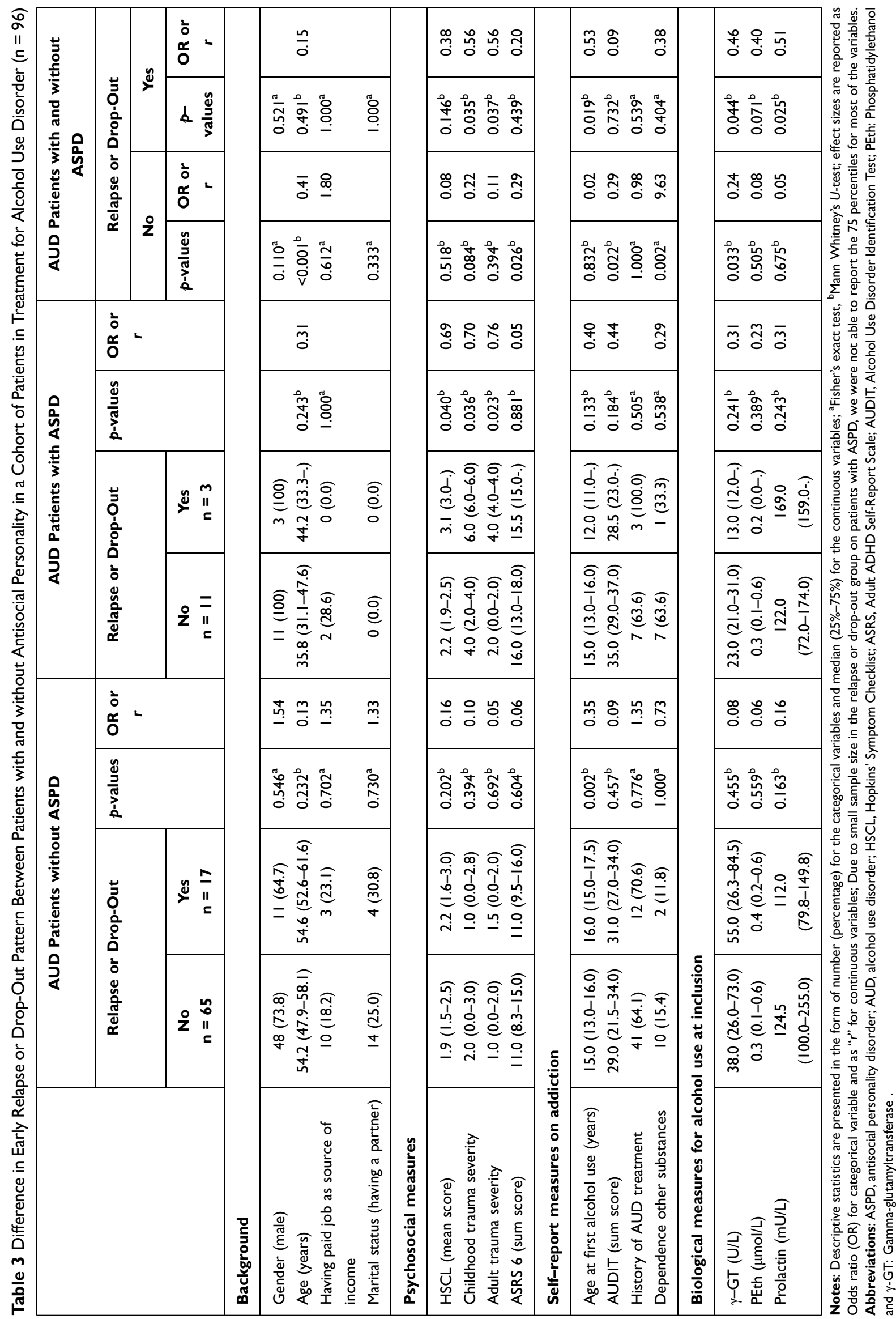



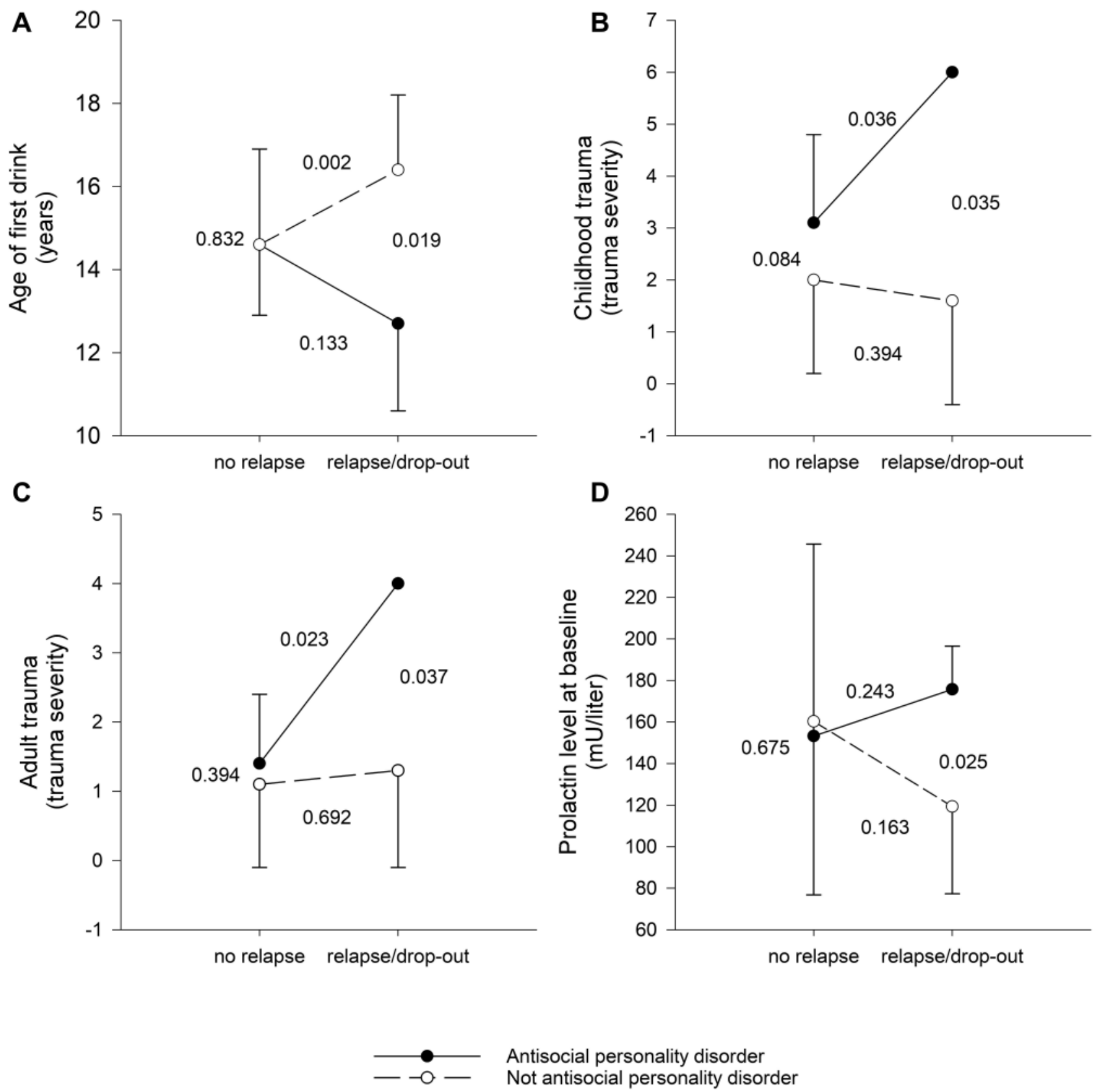

Figure I Comparison of alcohol use disorder patients $(\mathrm{N}=96)$ with and without comorbid antisocial personality in terms of early relapse or drop-out versus treatment adherence (No relapse and no drop-out) using Mann Whitney's U-test. Panel (A) shows age at first drink, panel (B) shows childhood trauma severity, panel (C) shows adult trauma severity and panel (D) shows baseline levels of prolactin using mean, standard deviation, and level of significance.

personality disorder in the AUD population is an important but difficult to treat combination that deserves special attention.

Many studies corroborate that comorbid ASPD complicates the treatment of AUD. ${ }^{38,64}$ In our study, however, the relapse or drop-out rate did not differ between patients with and without ASPD. Other studies have also demonstrated equal benefits from the alcohol treatment in patients with or without ASPD. ${ }^{65}$ The discrepant findings might be due to varying time points in the measurement of treatment outcome or different ways of defining treatment outcome. However, different factors seemed to predict relapse or drop-out in the two different groups.

Relapse or drop-out among the AUD patients with ASPD was associated with more lifetime trauma. This is partly in line with the finding of Farley et al that relapse in 
general is associated with a history of trauma. ${ }^{66} \mathrm{We}$ found that among those who relapsed or dropped out, the patients with ASPD had an earlier drinking debut. Early age of drinking debut is characteristic of ASPD ${ }^{67}$ In addition, Hingson et al found that younger age of drinking debut was associated with chronic relapsing alcohol dependence. ${ }^{68}$ We found that among the patients who relapsed or dropped out, those with ASPD had higher baseline prolactin levels. Literature suggests that AUD patients with ASPD have a heightened dopamine level which is related to decreased prolactin levels. ${ }^{69}$ One possible explanation for our contradictory finding of higher prolactin level in patients with ASPD who relapsed could be a tendency of these patients to uphold their dopamine homeostasis. In other words, ASPD patients with higher prolactin levels relapse possibly because they are biologically driven to rebound to their conventional state of being high in dopamine. This biological drive might also explain our finding of earlier drinking debut among ASPD patients who relapse compared to other AUD patients.

This is a medium-sized real-life study of patients in treatment for AUD involving a comprehensive investigation of background variables, factors related to vulnerability, but also clinically important issues and biochemical markers including those related to AUD. Despite its size, the study saw only a few relapses or drop-outs in the observation period, making the study of patients with ASPD vulnerable for under-powering and type II statistical errors. Caution should be used in interpreting the findings. The lower number of relapses or drop-out in the study may be due to the patients being treated in residential treatment clinics. Also, relapse or drop-out was measured early. Factors related to relapse and drop-out were a major focus of our study, but we had no direct assessment of the patients' motivation to stay sober and stay in treatment. There could be other barriers to treatment retention not measured in our study, including financial problems, social support, quality of treatment, and staff connection issues.

This study implies a need to acknowledge the presence of comorbid ASPD in AUD patients as these individuals differ on a number of measures compared to other AUD patients. Moreover, identification of comorbid ASPD may be useful in planning relapse prevention. Additional relapse prevention techniques could be tailored to meet the specific requirements of patients with ASPD. Lastly, higher biological drive towards relapse in patients with ASPD suggests that the use of cognitive and behavioral interventions alone may not suffice to prevent relapse in this group. Further studies on large sample sizes are recommended to confirm our preliminary findings on factors related to relapse or drop-out in AUD patients with ASPD.

\section{Conclusion}

In this study of AUD patients in treatment, $15 \%$ met the criteria for an antisocial personality disorder. These patients were male, of younger age, reported higher childhood trauma severity and more hazardous drinking behavior, and they more often had other substance dependence. There was no increased risk of early relapse or drop-out rate among the patients with ASPD, but they had specific predictors of early relapse or drop-out, including earlier drinking debut and more life-time trauma. Also, they seemed to be more "biologically driven" to drinking, as indicated by their increased prolactin levels.

\section{Abbreviations}

AUD, Alcohol Use Disorder; ASPD, Antisocial Personality Disorder; ADHD, Attention Deficit Hyperactivity Disorder; ASRS, Adult ADHD Self-Report Questionnaire; M.I.N.I, Mini International Neuropsychiatric Interview; AUDIT, Alcohol Use Disorders Identification test; HSCL-10, Hopkins Symptom checklist-10; $\gamma$-GT, Gamma-glutamyltransferase; PEth, Phosphatidylethanol; DSM-IV-TR, Diagnostic and Statistical Manual of Mental Disorders fourth edition text revision; DALYs, Disability-Adjusted Life Years; SFC-MS, Supercritical Fluid ChromatographyMass Spectrometry; UPC ${ }^{2}$, Ultraperformance Convergence Chromatography.

\section{Acknowledgments}

We would like to acknowledge the Research Council of Norway for funding the study; patients for participating in the study; and the treatment centres Riisby, Blå Kors and Trasoppklinikken for serving as data collection sites.

\section{Author Contributions}

All authors made a significant contribution to the work reported, whether that is in the conception, study design, execution, acquisition of data, analysis, and interpretation, or in all these areas; took part in drafting, revising or critically reviewing the article; gave final approval of the version to be published; have agreed on the journal to which the article has been submitted; and agree to be accountable for all aspects of the work. 


\section{Funding}

The study was funded by the Research Council of Norway. The funding body was not involved in the design of the study, the collection, analysis, or interpretation of data, or in the preparation of manuscript.

\section{Disclosure}

The authors report no conflicts of interest in this work.

\section{References}

1. World Health Organization. Harmful use of alcohol kills more than 3 million people each year, most of them men [press release]; 2018. Available from: https://www.who.int/news/item/21-09-2018-harmfuluse-of-alcohol-kills-more-than-3-million-people-each-year-most-ofthem-men\#: :text=of\%20them $\% 20$ men-,Harmful $\% 20 \mathrm{use} \% 20 \mathrm{of} \%$ 20alcohol\%20kills\%20more\%20than $\% 203 \% 20$ million,year $\% 2 \mathrm{C} \%$ $20 \mathrm{most} \% 20$ of $\% 20$ them $\% 20$ men\&text $=$ More $\% 20$ than $\% 203 \% 20 \mathrm{mil}$ lion\%20people \%20died $\% 20$ as\%20a\%20result $\% 20$ of,represents $\%$ 201\%20in\%2020\%20deaths.

2. Griswold MG, Fullman N, Hawley C, et al. Alcohol use and burden for 195 countries and territories, 1990-2016: a systematic analysis for the global burden of disease study 2016. The Lancet. 2018;392 (10152):1015-1035. doi:10.1016/S0140-6736(18)31310-2

3. Institute of Public Health. Substance use disorders in Norway public health report - health status in Norway [online document]; 2016. Available from: https://www.fhi.no/en/op/hin/mental-health/intoxi cants-and-substance-use/. Accessed April 01, 2021.

4. Torvik FA, Ystrom E, Gustavson K, et al. Diagnostic and genetic overlap of three common mental disorders in structured interviews and health registries. Acta Psychiatr Scand. 2018;137(1):54-64. doi:10.1111/acps.12829

5. Donoghue K, Elzerbi C, Saunders R, Whittington C, Pilling S, Drummond C. The efficacy of acamprosate and naltrexone in the treatment of alcohol dependence, Europe versus the rest of the world: a meta-analysis. Addiction. 2015;110(6):920-930. doi:10.1111/add.12875

6. Tanner-Smith EE, Lipsey MW. Brief alcohol interventions for adolescents and young adults: a systematic review and meta-analysis. J Subst Abuse Treat. 2015;51:1-18. doi:10.1016/j.jsat.2014.09.001

7. Moos RH, Moos BS. Rates and predictors of relapse after natural and treated remission from alcohol use disorders. Addiction. 2006;101 (2):212-222. doi:10.1111/j.1360-0443.2006.01310.x

8. Dawson DA, Grant BF, Stinson FS, Chou PS. Estimating the effect of help-seeking on achieving recovery from alcohol dependence. Addiction. 2006;101(6):824-834. doi:10.1111/j.1360-0443.2006.01433.x

9. Rehm J, Allamani A, Elekes Z, et al. Alcohol dependence and treatment utilization in Europe- a representative cross-sectional study in primary care. BMC Fam Pract. 2015;16(1):90. doi:10.1186/s12875-015-0308-8

10. Durazzo TC, Meyerhoff DJ. Psychiatric, demographic, and brain morphological predictors of relapse after treatment for an alcohol use disorder. Alcohol Clin Exp Res. 2017;41(1):107-116. doi:10.1111/acer.13267

11. Nguyen L-C, Durazzo TC, Dwyer CL, et al. Predicting relapse after alcohol use disorder treatment in a high-risk cohort: the roles of anhedonia and smoking. $J$ Psychiatr Res. 2020;126:1-7. doi:10.1016/j.jpsychires.2020.04.003

12. Walitzer KS, Dearing RL. Gender differences in alcohol and substance use relapse. Clin Psychol Rev. 2006;26(2):128-148. doi:10.1016/j.cpr.2005.11.003

13. Heffner JL, Blom TJ, Anthenelli RM. Gender differences in trauma history and symptoms as predictors of relapse to alcohol and drug use. Am $J$ Addict. 2011;20(4):307-311. doi:10.1111/j.15210391.2011.00141.x
14. Engel K, Schaefer M, Stickel A, Binder H, Heinz A, Richter C. The role of psychological distress in relapse prevention of alcohol addiction. Can high scores on the SCL-90-R predict alcohol relapse? Alcohol Alcohol. 2016;51(1):27-31. doi:10.1093/alcalc/agv062

15. Oliva F, Nibbio G, Vizzuso P, et al. Gender differences in anxiety and depression before and after alcohol detoxification: anxiety and depression as gender-related predictors of relapse. Eur Addict Res. 2018;24:163-172. doi:10.1159/000490046

16. Wilens TE, Adler LA, Tanaka Y, et al. Correlates of alcohol use in adults with ADHD and comorbid alcohol use disorders: exploratory analysis of a placebo-controlled trial of atomoxetine. Curr Med Res Opin. 2011;27(12):2309-2320. doi:10.1185/03007995.2011.628648

17. Walter H, Ramskogler K, Semler B, Lesch OM, Platz W. Dopamine and alcohol relapse: D1 and D2 antagonists increase relapse rates in animal studies and in clinical trials. J Biomed Sci. 2001;8(1):83-88. doi:10.1007/BF02255975

18. Liu Y, Jean-Richard-dit-Bressel P, Yau JO, et al. The mesolimbic dopamine signatures of relapse to alcohol-seeking. J Neurosci. 2020;40:6409-6427.

19. Ben-Jonathan N, Hnasko R. Dopamine as a prolactin (PRL) inhibitor. Endocr Rev. 2001;22(6):724-763.

20. Hillemacher T, Bayerlein K, Wilhelm J, et al. Prolactin serum levels and alcohol craving-an analysis using Lesch's typology. Neuropsychobiology. 2006;53(3):133-136. doi:10.1159/000092543

21. Stohs ME, Schneekloth TD, Geske JR, Biernacka JM, Karpyak VM. Alcohol craving predicts relapse after residential addiction treatment. Alcohol Alcohol. 2019;54(2):167-172. doi:10.1093/alcalc/agy093

22. Brorson HH, Arnevik EA, Rand-Hendriksen K, Duckert F. Drop-out from addiction treatment: a systematic review of risk factors. Clin Psychol Rev. 2013;33(8):1010-1024. doi:10.1016/j.cpr.2013.07.007

23. Deane FP, Wootton DJ, Hsu CI, Kelly PJ. Predicting dropout in the first 3 months of 12-step residential drug and alcohol treatment in an Australian sample. J Stud Alcohol Drugs. 2012;73(2):216-225. doi:10.15288/jsad.2012.73.216

24. Coriale G, Fiorentino D, De Rosa F, et al. Treatment of alcohol use disorder from a psychological point of view. Riv Psichiatr. 2018;53 (3):141-148. doi:10.1708/2925.29416

25. Foulds J, Newton-Howes G, Guy NH, Boden JM, Mulder RT. Dimensional personality traits and alcohol treatment outcome: a systematic review and meta-analysis. Addiction. 2017;112 (8):1345-1357. doi:10.1111/add.13810

26. Zandberg LJ, Rosenfield D, Alpert E, McLean CP, Foa EB. Predictors of dropout in concurrent treatment of posttraumatic stress disorder and alcohol dependence: rate of improvement matters. Behav Res Ther. 2016;80:1-9. doi:10.1016/j.brat.2016.02.005

27. Brorson HH, Arnevik EA, Rand K. Predicting dropout from inpatient substance use disorder treatment: a prospective validation study of the OQ-analyst. Subst Abuse. 2019;13:1178221819866181. doi:10.1177/1178221819866181

28. Leggio L, Kenna GA, Fenton M, Bonenfant E, Swift RM. Typologies of alcohol dependence. From Jellinek to genetics and beyond. Neuropsychol Rev. 2009;19(1):115-129. doi:10.1007/s11065-0089080-z

29. Helle AC, Watts AL, Trull TJ, Sher KJ. Alcohol use disorder and antisocial and borderline personality disorders. Alcohol Res. 2019;40 (1). doi:10.35946/arcr.v40.1.05

30. American Psychiatric Association. Diagnostic and Statistical Manual of Mental Disorders. 4th ed. Washington, DC: American Psychiatric Association; 2000.

31. Trull TJ, Jahng S, Tomko RL, Wood PK, Sher KJ. Revised NESARC personality disorder diagnoses: gender, prevalence, and comorbidity with substance dependence disorders. J Pers Disord. 2010;24 (4):412-426. doi:10.1521/pedi.2010.24.4.412

32. Sheehan L, Nieweglowski K, Corrigan P. The stigma of personality disorders. Curr Psychiatry Rep. 2016;18(1):11. doi:10.1007/s11920015-0654-1 
33. Goldstein RB, Dawson DA, Grant BF. Antisocial behavioral syndromes in adulthood and alcohol use disorder treatment over three-year follow-up: results from wave 2 of the national epidemiologic survey on alcohol and related conditions. J Am Psychiatr Nurses Assoc. 2010;16(4):212-226. doi:10.1177/1078390310375846

34. Guy N, Newton-Howes G, Ford H, Williman J, Foulds J. The prevalence of comorbid alcohol use disorder in the presence of personality disorder: systematic review and explanatory modelling. Personal Ment Health. 2018;12(3):216-228. doi:10.1002/pmh.1415

35. Rosenström T, Torvik FA, Ystrom E, et al. Prediction of alcohol use disorder using personality disorder traits: a twin study. Addiction. 2018;113(1):15-24. doi:10.1111/add.13951

36. Brennan GM, Hyde LW, Baskin-Sommers AR. Antisocial pathways associated with substance use disorders: characterizing etiological underpinnings and implications for treatment. Curr Opin Behav Sci. 2017;13:124-129. doi:10.1016/j.cobeha.2016.11.014

37. Goldstein RB, Dawson DA, Saha TD, Ruan WJ, Compton WM, Grant BF. Antisocial behavioral syndromes and DSM-IV alcohol use disorders: results from the national epidemiologic survey on alcohol and related conditions. Alcohol Clin Exp Res. 2007;31 (5):814-828. doi:10.1111/j.1530-0277.2007.00364.x

38. Kranzler HR, Boca FKD, Rounsaville BJ. Comorbid psychiatric diagnosis predicts three-year outcomes in alcoholics: a posttreatment natural history study. J Stud Alcohol. 1996;57 (6):619-626. doi:10.15288/jsa.1996.57.619

39. Zikos E, Gill KJ, Charney DA. Personality disorders among alcoholic outpatients: prevalence and course in treatment. Can J Psychiatry. 2010;55(2):65-73. doi:10.1177/070674371005500202

40. Sargeant MN, Bornovalova MA, Trotman AJM, Fishman S, Lejuez CW. Facets of impulsivity in the relationship between antisocial personality and abstinence. Addict Behav. 2012;37(3):293-298. doi:10.1016/j.addbeh.2011.11.012

41. Martinez P, Neupane SP, Perlestenbakken B, Toutoungi C, Bramness JG. The association between alcohol use and depressive symptoms across socioeconomic status among 40- and 45-year-old Norwegian adults. BMC Public Health. 2015;15(1):1146. doi:10.1186/s12889-015-2479-6

42. Strand BH, Dalgard OS, Tambs K, Rognerud M. Measuring the mental health status of the Norwegian population: a comparison of the instruments SCL-25, SCL-10, SCL-5 and MHI-5 (SF-36). Nord J Psychiatry. 2003;57(2):113-118. doi:10.1080/08039480310000932

43. Kessler RC, Adler L, Ames M, et al. The World Health Organization adult ADHD self-report scale (ASRS): a short screening scale for use in the general population. Psychol Med. 2005;35(2):245. doi:10.1017/ S0033291704002892

44. Kessler RC, Adler LA, Gruber MJ, Sarawate CA, Spencer T, Van Brunt DL. Validity of the World Health Organization adult ADHD self-report scale (ASRS) screener in a representative sample of health plan members. Int J Methods Psychiatr Res. 2007;16(2):52-65. doi:10.1002/mpr.208

45. Oerbeck B, Overgaard K, Pripp AH, Aase H, ReichbornKjennerud T, Zeiner P. Adult ADHD symptoms and satisfaction with life: does age and sex matter? J Atten Disord. 2019;23 (1):3-11. doi:10.1177/1087054715612257

46. World Health Organization. AUDIT: the Alcohol Use Disorders Identification Test: guidelines for use in primary health care/ Thomas F. Babor ... [et al.], 2nd ed. Geneva: World Health Organization; 2001.

47. Erevik EK, Pallesen S, Vedaa Ø, Andreassen CS, Torsheim T. Alcohol use among Norwegian students: demographics, personality and psychological health correlates of drinking patterns. Nord Stud Alcohol Drugs. 2017;34(5):415-429. doi:10.1177/1455072517709918

48. Hahn JA, Anton RF, Javors MA. The formation, elimination, interpretation, and future research needs of phosphatidylethanol for research studies and clinical practice. Alcohol Clin Exp Res. 2016;40(11):2292-2295. doi:10.1111/acer.13213
49. Gnann H, Weinmann W, Thierauf A. Formation of phosphatidylethanol and its subsequent elimination during an extensive drinking experiment over 5 days. Alcohol Clin Exp Res. 2012;36 (9):1507-1511. doi:10.1111/j.1530-0277.2012.01768.x

50. Ulwelling W, Smith K. The PEth blood test in the security environment: what it is; why it is important; and interpretative guidelines. J Forensic Sci. 2018;63(6):1634-1640. doi:10.1111/1556-4029.13874

51. Şimşek M, Dinç M, Ögel K. Determinants of the addiction treatment drop-out rates in an addiction counseling centre: a cross-sectional study. Psychiatr Clin Psychopharmacol. 2019;29(4):446-454. doi:10.1080/24750573.2018.1505283

52. Fritz CO, Morris PE, Richler JJ. Effect size estimates: current use, calculations, and interpretation. J Exp Psychol Gen. 2012;141 (1):2-18. doi:10.1037/a0024338

53. Compton WM, Conway KP, Stinson FS, Colliver JD, Grant BF. Prevalence, correlates, and comorbidity of DSM-IV antisocial personality syndromes and alcohol and specific drug use disorders in the United States: results from the national epidemiologic survey on alcohol and related conditions. J Clin Psychiatry. 2005;66 (6):677-685. doi:10.4088/JCP.v66n0602

54. Cloninger CR, Sigvardsson S, Bohman M. Type I and type II alcoholism: an update. Alcohol Health Res World. 1996;20(1):18-23.

55. Moss HB, Chen CM, Yi HY. Subtypes of alcohol dependence in a nationally representative sample. Drug Alcohol Depend. 2007;91 (2-3):149-158. doi:10.1016/j.drugalcdep.2007.05.016

56. Murray MG, Anthenelli RM, Maxwell RA. Use of health services by men with and without antisocial personality disorder who are alcohol dependent. Psychiatr Serv. 2000;51(3):380-382. doi:10.1176/appi. ps.51.3.380

57. Loh E, Chow J. Assessment of symptoms of attention-deficit hyperactivity disorder in adults with substance use disorders. Psychiatr Serv. 1997;48(11):1379. doi:10.1176/ps.48.11.1378

58. Estévez N, Dey M, Eich-Höchli D, Foster S, Gmel G, MohlerKuo M. Adult attention-deficit/hyperactivity disorder and its association with substance use and substance use disorders in young men. Epidemiol Psychiatr Sci. 2016;25(3):255-266. doi:10.1017/ S2045796015000360

59. DeLisi M, Drury AJ, Elbert MJ. The etiology of antisocial personality disorder: the differential roles of adverse childhood experiences and childhood psychopathology. Compr Psychiatry. 2019;92:1-6. doi:10.1016/j.comppsych.2019.04.001

60. Enoch M-A, Albaugh BJ. Review: genetic and environmental risk factors for alcohol use disorders in American Indians and Alaskan natives. Am J Addict. 2017;26(5):461-468. doi:10.1111/ajad.12420

61. Hesselbrock VM, Hesselbrock MN. Are there empirically supported and clinically useful subtypes of alcohol dependence? Addiction. 2006;101:98. doi:10.1111/j.1360-0443.2006.01596.x

62. Creswell KG, Bachrach RL, Wright AGC, Pinto A, Ansell E. Predicting problematic alcohol use with the DSM-5 alternative model of personality pathology. Personal Disord. 2016;7 (1):103-111. doi:10.1037/per0000131

63. Zucker RA, Ellis DA, Fitzgerald HE, Bingham CR, Sanford K. Other evidence for at least two alcoholisms II: life course variation in antisociality and heterogeneity of alcoholic outcome. Dev Psychopathol. 1996;8(4):831-848. doi:10.1017/S0954579400007458

64. Rounsaville BJ, Dolinsky ZS, Babor TF, Meyer RE. Psychopathology as a predictor of treatment outcome in alcoholics. Arch Gen Psychiatry. 1987;44(6):505-513. doi:10.1001/archpsyc.1987.01800180015002

65. Newton-Howes GM, Foulds JA, Guy NH, Boden JM, Mulder RT. Personality disorder and alcohol treatment outcome: systematic review and meta-analysis. Br J Psychiatry. 2017;211(1):22-30. doi:10.1192/bjp.bp.116.194720

66. Farley M, Golding JM, Young G, Mulligan M, Minkoff JR. Trauma history and relapse probability among patients seeking substance abuse treatment. J Subst Abuse Treat. 2004;27(2):161-167. doi:10.1016/j.jsat.2004.06.006 
67. Westermeyer J, Thuras P. Association of antisocial personality disorder and substance disorder morbidity in a clinical sample. $A m$ J Drug Alcohol Abuse. 2005;31(1):93-110. doi:10.1081/ADA-47895

68. Hingson RW, Heeren T, Winter MR. Age at drinking onset and alcohol dependence: age at onset, duration, and severity. Arch Pediatr Adolesc Med. 2006;160(7):739-746. doi:10.1001/ archpedi.160.7.739
69. Kolla NJ, Wang CC. Chapter 29 - alcohol and violence in psychopathy and antisocial personality disorder: neural mechanisms. In: Preedy VR, editor. Neuroscience of Alcohol. Academic Press; 2019:277-285.

\section{Publish your work in this journal}

Substance Abuse and Rehabilitation is an international, peerreviewed, open access journal publishing original research, case reports, editorials, reviews and commentaries on all areas of addiction and substance abuse and options for treatment and rehabilitation. The manuscript management system is completely online and includes a very quick and fair peer-review system. Visit http://www.dovepress. com/testimonials.php to read real quotes from published authors. 